\title{
Can Moxibustion Treatment Improve Vertical Jump Biomechanics?
}

\author{
Yuwei Liu' ${ }^{1}$, Kyle Finnie ${ }^{2}$ and Shirui Shao ${ }^{1,3^{*}}$ \\ ${ }^{1}$ Faculty of Sports Science, Ningbo University, China \\ ${ }^{2}$ University of the West of Scotland, UK \\ ${ }^{3}$ Department of Mechanical Engineering, Eotvos Lorand University, Hungary \\ * corresponding author: shaoshiruinb@qq.com
}

\section{Keywords: Moxibustion; Vertical jump; Biomechanics}

\begin{abstract}
Moxibustion has been known by more and more people as a traditional Chinese treatment technique. Although the mechanism of moxibustion is not clear, the therapeutic effect of moxibustion is obvious to all. The purpose of this paper is to investigate whether moxibustion can alleviate the fatigue state after the movement of human body by means of biomechanics testing. Therefore, analysis of hip, knee and ankle kinematics during forefoot landing from a vertical jump and comparison of moxibustion treatment versus no treatment could identify further evidence of its practicality when dealing with exercise induced fatigue. The joint angle and the angular velocity of the joint were obtained from Vicon motion anakysis system, and peak vGRF and time was measure with Kistler. Overall, it can be concluded that moxibustion can have a direct effect on a UK citizens vertical jump lower limb kinematics during a fatigued state. This was particularly evident within the significant differences between peak knee angle during the take-off phase and peak ankle angle during the take-off and landing phases. Furthermore, statistically significant differences between the vertical jumps angular velocity of the knee and hip joints provide further evidence that moxibustion treatments physiological adaptations can alter and improve vertical jump biomechanics. Moreover, not only did moxibustion treatment improve peak angles and angular velocity, statistically significant differences in jump time were evident providing further evidence that moxibustion can in fact improve fatigued individuals jumping kinematics, but there is no significant different in peak vGRF. Evidence from these results could perhaps suggest that moxibustion could be used as an injury preventative measure to reduce fatigue and aid performance. Further investigation with a larger sample size and perhaps an alternative control is required to further validate these findings.
\end{abstract}

\section{Introduction}

Exercise-induced fatigue is the decline in ability of the bodily physiological process to sustain certain levels of functioning and/or maintain an expected exercise intensity. This suggests that athletes may experience fatigue after intensive physical training or during vigorous matches decreasing recovery ability and resulting in delayed onset of muscle soreness [1]. A reduction of neuromuscular ability is of main concern regarding exercise-induced fatigue which in turn can increase the likelihood of injury [2]. This is particularly evident with regards to biomechanical analysis of safe landing mechanics with high instances of knee and ankle injuries. Safe landing entails landing efficiently to absorb shock through the hip knee and ankle joints and allowing the body to rebound safely and powerfully. Fong et al. [3] discovered that an increase in ankle plantar-flexor extensibility and dorsiflexion range of motion may help to reduce injury risk. This type of research has led to the development and evolution of preventative and recovery strategies to combat fatigue. With many athletes enduring heavy schedules their reliance on recovery strategies is huge therefore these strategies such as specified training tools, medical interventions and ergogenic aids continue to develop.

However, in China ancient Chinese medicine remedies such as acupuncture, cupping and moxibustion are often preferred to more modern strategies. These have played a remarkable role within Chinese athlete's success in the last 20 years. Many research articles have investigated the effects these strategies can have when combined. However, there is very limited evidence of moxibustion being used 
alone [4]. Moxibustion is a traditional Chinese method that is "a therapeutic heat that permeates the skin and affects the flow of "qi" (energy) and blood in the area being treated". This method uses heat and smoke generated by burning herbal blocks containing "Artemisia vulgaris" (Mugwort) to stimulate blood flow to deep tissues through the meridian acupoints [5]. It can be administered directly by burning or piercing the skin or indirectly by radiating the heat around the acupoint without damaging the skin. Shen et al. [6] state that direct moxibustion may produce more potent therapeutic effects by thermal action, whilst indirect moxibustion produces a less modest thermal action. Li et al. [7] found that combined moxibustion with cupping was effective in reducing fatigue in gymnasts. Results indicated that mild moxibustion and cupping can help to elevate creatine kinase level during induced exhaustive exercise. This increase in creatine kinase levels allows for more ATP to be synthesised thus, eliminating lactate built up from exhaustive exercise that can help to reduce fatigue. In agreement, Kim et al. [8] discovered that moxibustion treatment can significantly lower fatigue severity score compared with a control group. Outside of the sports industry, moxibustion has been used as an intervention to treat cancer related fatigue. He, Wang and $\mathrm{Li}[9]$ discovered when moxibustion plus routine care was administered together, the combination had a significant benefit in improving severe fatigue. This would suggest that moxibustion is not only effective at combatting fatigue within the sports industry but is highly relevant in modern clinical healthcare.

In terms of biomechanics analysis of moxibustion treatment alone, no articles exist. On the other hand, there are an abundance of papers regarding worldwide use of acupuncture and biomechanical analysis. Analysis of spinal structure pathology and disk degeneration from acupuncture treatment discovered significant improvements compared with a control group [10]. Also, Zhang [11]found that acupuncture significantly improved single step walking speed in stroke patients. This evidence further suggests that ancient Chinese medicine techniques can have a positive impact not only on improving spinal mechanics but also lower limb biomechanics. With regards to lower limb mechanics, safe landing technique has become a focal point of interest. Borotikar et al. [12] state that induced fatigue created significant increases in initial contact hip extension and internal rotation, increased peak stance knee abduction and internal rotation and increased ankle supination angles when landing. This discovery concludes that exercise induced fatigue is a huge factor regarding increased injury risk when landing, especially non-contact anterior cruciate ligament damage. By correlating these findings together perhaps a link between ancient Chinese medical treatments and lower limb kinematics through vertical jumping could be made. In particular moxibustion treatment due to its limited research within the biomechanics field. The physiological adaptations from moxibustion could perhaps act to reduce post exercise fatigue and decrease injury risk thus, improving jumping kinematics.

\section{Methods}

The 8-camera Vicon motion analysis system (Oxford Metrics Ltd., Oxford, UK) operating at $100 \mathrm{~Hz}$ was positioned around the participant to record the vertical jump lower limb landing kinematics. Sixteen retro reflective markers were attached to the participants' lower limb in accordance with the standard Plug-In-Gait model. A force platform (Kistler, Switzerland) was used to capture the ground reaction force landing data from the participants left leg. Moxibustion therapy stick burner was used for the twenty-minute suspended moxibustion treatment phase. Suspended moxibustion technique was used due to it being a non-invasive procedure. A Keiser spin bike (California, USA) was used for the exercise element of testing. The experiments all took place in the biomechanics research laboratory at Ningbo University. Three data collectors were present and performed the same duties throughout testing to ensure validity. The Vicon Nexus Motion Analysis System was calibrated by waving the calibration wand in a figure of eight around the test area to allow for a clear unobstructed field view for the camera system. Static calibration was done by placing the calibration wand on the GRF plates to set the origin. The client wore tight fitting compression shorts to make marker placement and measurements accessible. Anthropometric measures (height, weight, leg length, ankle width and knee width) of was recorded to create a client specific profile. Sixteen reflective markers in total were attached in accordance to Plug-in-Gait marker placement to enable detection of the participants lower limb movement using the 
Vicon Motion Analysis system. The participant stood with his left foot on the GRF plate and held a static stance to allow for camera recognition.

Test two was performed forty-eight hours later to ensure the client was fully recovered from test one. The participant followed the same diet and hydration plan as the previous test and laboratory conditions were made as similar as possible. Once again, eight baseline non-exercise vertical jumps were performed with GRF, hip, knee and ankle kinematic data recorded. The same Keiser spin bike was used for a 5-minute warm up. Once again, the participant cycled at 50 watts for 1 minute with increments of 25 watts for each minute thereafter with cadence self-selected throughout. The duration of the test was until the participant was fully fatigued and their finishing wattage was noted. RPE (1-20 scale) was used to ensure the client was truly working at their maximum workload. The participant was then subjected to 20-min of suspended moxibustion treatment to the lower limbs from qualified Chinese medicine students. Suspended moxibustion treatment was applied to the hip (GB-30), knee (GB-33) and ankle (GB-40) gall bladder meridian acupoints in a waving motion [13]. After the moxibustion treatment the participant performed eight vertical jumps on the command of "jump" from the data collector. Ankle, knee and hip angle data and ground reaction force data was recorded to aid as a comparison measure.

The Vicon Nexus 1.8.5 system recorded the data for kinematics and GRF. Left leg vertical jump peak ankle, knee and hip joint angles, angular jump velocity, jump time data and peak vGRF was transferred to Microsoft Excel 2016 for Windows 10. Peak angle data from the sagittal plane was split into phases, average joint angle velocity and average jump time and standard deviations were calculated and displayed. Paired samples t-test (SPSS 18, IBM, Hampshire, UK) were used to determine the difference in means between baseline jump and treatments and no treatment versus moxibustion treatment peak angles within take-off and landing phases. Level of significance was set at 0.05 and result figures were created using Microsoft Excel 2016 software.

\section{Results}

Table 1 Average peak values and standard deviations for left limb joints split into take-off and landing jump phases during the sagittal plane of motion. $(M \pm S D)$

Sagittal Plane Jump $\quad$ Max Hip Angle L $\left(^{\circ}\right) \quad$ Max Knee Angle L $\left(^{\circ}\right) \quad$ Max Ankle Angle L $\left(^{\circ}\right)$

\begin{tabular}{lllll}
\hline Take-off & 1 Baseline & $73.13 \pm 2.36$ & $66.39 \pm 3.34(*)$ & $24.31 \pm 3.07$ \\
& 2No treatment & $83.86 \pm 4.91$ & $59.78 \pm 6.29(*)(* *)$ & $18.70 \pm 1.25(* *)$ \\
& 3 Baseline & $79.97 \pm 0.62$ & $80.31 \pm 2.79$ & $21.74 \pm 2.79$ \\
Landing & 4 Moxibustion & $79.71 \pm 1.94$ & $85.91 \pm 4.13(* *)$ & $22.46 \pm 0.59(* *)$ \\
& 1 Baseline & $65.20 \pm 12.48$ & $51.62 \pm 3.05$ & $24.70 \pm 0.75(*)$ \\
& 2No treatment & $77.06 \pm 6.03$ & $51.89 \pm 3.32$ & $16.58 \pm 0.70\left(^{*}\right)(* *)$ \\
& 3 Baseline & $72.21 \pm 3.02(*)$ & $51.45 \pm 2.57$ & $24.42 \pm 0.95$ \\
& 4 Moxibustion & $76.47 \pm 2.70(*)$ & $57.64 \pm 9.98$ & $24.09 \pm 1.40(* *)$
\end{tabular}

Note: $*(P<0.05)$ donates a significant difference between jumps and ** $(P<0.05)$ donates a significant difference between treatments. 


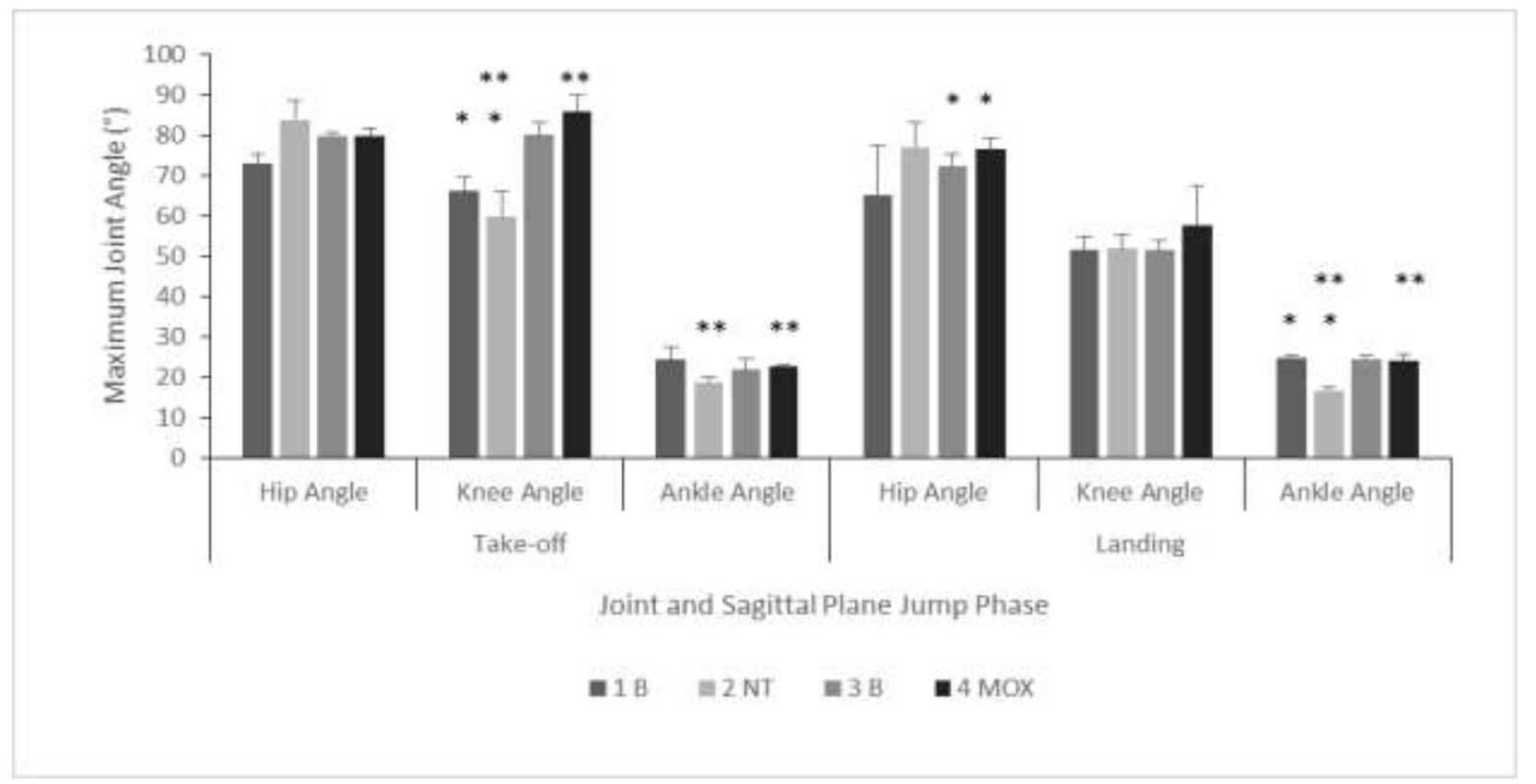

Figure 1. Comparison of sagittal plane left limb peak joint angles between take-off and landing jump phases.

Note: $(B=$ Baseline, $N T=$ No Treatment, $M O X=$ Moxibustion Treatment $) . P<0.05(*)$ indicates significant differences between jumps and $P<0.05\left(^{* *}\right)$ indicates significant differences between treatments.

\section{Discussion}

Within the take-off phase significant findings were evident in Table 1 and Figure 1 . However, within peak hip angles no significant differences were discovered $(\mathrm{P}>0.05)$. This may suggest that both exercise and moxibustion have no effect on hip joint angles during the take-off phase of a vertical jump. Similarly, Benjaminse et al. [14] found that hip angles did not change during a single leg-stop jump exercise despite being in a fatigued state. On the other hand, significant differences were discovered within the peak left knee joints during take-off between jumps 1 and $2(\mathrm{P}=0.041)$ and between no treatment and moxibustion treatment $(\mathrm{P}=0.014)$. As expected knee flexion is higher within the take-off phase of a vertical jump due to the jumper having to overcome inertia to leave the ground [15]. This data would suggest that fatigue had an adverse effect on the knee joint during the first test causing knee joint flexibility to reduce. The significant difference between knee non-treatment versus moxibustion treatment suggests that despite being in a fatigued state moxibustion alone can increase knee flexibility during the take-off phase. The physiological adaptations from moxibustion treatment in turn can allow for greater force to be generated allowing for the jumper to improve jump time and velocity. Peak knee angle did not show any significant differences $(\mathrm{P}=0.894, \mathrm{P}=0.309$ and $\mathrm{P}=0.437)$ between each jump or between treatments suggesting that knee angle was consistent throughout landing despite the use of a fatigue inducing exercise intervention or moxibustion therapy. This could perhaps be down to an increase in ankle flexibility thus reducing the knee flexion angle. Also, knee flexion during landing can be affected by trunk motion as previously mentioned [16]. However, Chappell et al. [17] found that knee joint flexion decreased during a stop-jump task when fatigued increasing injury risk. Perhaps by using the same testing process on a female different peak value would be observed due to valgus knee being more prominent in females. Finally, peak values within the ankle angle did show statistically significant differences between jumps 1 and jump $2(\mathrm{P}=0.001)$ and between non-treatment and moxibustion treatment $(\mathrm{P}=0.005)$. The statistical difference decrement between jumps 1 and 2 was highly significant $\mathrm{P}=0.001$, suggesting that fatigue has a highly detrimental effect on ankle landing angles. This further provides evidence to Kernozek et al. [2] statement that fatigued athletes are of a 
greater risk of injury. On the other hand, the significant difference increase between the treatments in peak ankle angle suggests that moxibustion treatment alone can have a positive effect on improving peak ankle angle dorsiflexion thus reducing the risk of injury [3].

Moxibustion treatment has been applied and believed to protect health in China for thousands of years [18]. Within the sagittal plane angular velocity of the knee joint significant statistical differences were discovered between baseline and no treatment groups, $\mathrm{P}=0.045$. No treatment and moxibustion also show significant statistical differences, $\mathrm{P}=0.010$. Comparison of the baseline and moxibustion treatment groups found highly statistically significant differences $\mathrm{P}=0.000$ within angular velocity. There were significantly statistical differences between two groups at all measurements of the knee. This is in line with the original hypothesis. The angular velocity of the knee joint increased significantly in the moxibustion group, compared with the baseline and no treatment groups. This may also prove that moxibustion can improve the angular velocity of the knee joint during the longitudinal jump [19]. After the use of moxibustion, the recovery of the knee joint is better than that of the non-treatments group [20]. Therefore, it can be speculated that moxibustion can effectively restore the activity of the human knee joint. No statistically significant findings were discovered between baseline and treatment jumps $(\mathrm{P}=0.817$ and $\mathrm{P}=0.061)$. This data would suggest that the fatigue inducing exercise had very little effect on ground reaction force jump time. As the results from jump 1 baseline and jump 2 no treatment shows a slight decrease in time this would agree with the theory that fatigue can have a detrimental effect on performance [21]. There were significant differences discovered between the non-treatment and moxibustion treatment jump times $(\mathrm{P}=0.045)$. This result portrays that moxibustion therapy alone can significantly increase vertical jump time after fatigue inducing exercise. By correlating jump time data with peak angle data, it can be concluded that increased knee joint flexion during take-off following moxibustion therapy when in a fatigued state can help increase vertical jump time within a UK citizen. There was no difference in peak vGRF between baseline, moxibustion and no treatment in landing phrase $(\mathrm{P}=0.785, \mathrm{P}=0.859, \mathrm{P}=0.925)$. As Nathaniel et al.[22] also believes that peak vGRF does not have a significant difference between landing. Therefore, we can speculate that moxibustion has little effect on the peak vGRF in vertical jump within a UK citizen.

\section{References}

[1] M. Ding, X.S. Dong, X.H. Wang, X. Wang and X.J. Zhang. Effects of Suspended Moxibustion on Delayed Onset Muscle Soreness: A Randomized Controlled Double-Blind Pilot Study. Journal of Sports Science and Medicine. 16(2017). 203-208.

[2] T. Kernozek, M. Torry and M. Iwasaki. Gender Differences in Lower Extremity Landing Mechanics Caused by Neuromuscular Fatigue. The American Journal of Sports Medicine, 36(3), (2008).pp.554-565.

[3] C. Fong, J. Blackburn, M. Norcross, M. McGrath and D. Padua: Ankle-Dorsiflexion Range of Motion and Landing Biomechanics. Journal of Athletic Training, 46(1), (2011). pp.5-10.

[4] H. Deng and X. Shen.The Mechanism of Moxibustion: Ancient Theory and Modern Research. Evidence-Based Complementary and Alternative Medicine, 2013 (2013), pp.1-7.

[5] F. Cardini and H. Weixin. Moxibustion for Correction of Breech Presentation. JAMA, 280(18), (1998) p.1580.

[6] X. Shen, G. Ding, J. Wei, L. Zhao, Y. Zhou, H. Deng and L. Lao. An infrared radiation study of the biophysical characteristics of traditional moxibustion. Complementary Therapies in Medicine, 14(3), (2006) pp.213-219.

[7] D. Sun, Y. Zhang, D. Chen, A. Zhang, M. Xu, Z. Li, X. Zhu, H. Jiang, Y. Song, and W. Hao. Effect of moxibustion therapy plus cupping on exercise-induced fatigue in athletes. Journal of Acupuncture and Tuina Science, 10(5), (2012),pp.281-286.

[8] H. Kim, S. Yoo, H. Park, and C. Son. Indirect Moxibustion (CV4 and CV8) Ameliorates Chronic Fatigue: A Randomized, Double-Blind, Controlled Study. The Journal of Alternative and Complementary Medicine, 19(2), (2013),pp.134-140. 
[9] X. He, Q. Wang, and P. Li. Acupuncture and Moxibustion for Cancer-related Fatigue: a Systematic Review and Meta-analysis. Asian Pacific Journal of Cancer Prevention, 14(5), (2013),pp.3067-3074.

[10] M. Adams, N. Bogduk, K. Burton and P. Dolan. The Biomechanics of Back Pain. Physiotherapy (2002) 89 (2) pp.133-133

[11] Y. Zhang, H. Liu, C. Fu, Y. Ning, J. Zhang, L. Zhou, Z. Li, and P. Bai. The biomechanical effect of acupuncture for poststroke cavovarus foot: study protocol for a randomized controlled pilot trial. Trials, , (2016)17(1)

[12] B. Borotikar, R. Newcomer, R. Koppes and S. McLean. Combined effects of fatigue and decision making on female lower limb landing postures: Central and peripheral contributions to ACL injury risk. Clinical Biomechanics, (2008)23(1), pp.81-92.

[13] C.Wilmott . Food in Traditional Chinese Medicine .Chinese Journal of Integrative Medicine, vol. 24, (1987) pp. 25-37

[14] A. Benjaminse, A. Habu, T.C. Sell et al. Fatigue alters lower extremity kinematics during a single-leg stop-jump task. Knee Surgery, Sports Traumatology, Arthroscopy (2008) 16: pp400-407.

[15] N. P. Linthorne. Analysis of standing vertical jumps using a force platform, American Journal of Physics, 69 (2001), 1198-1204.

[16] J. Blackburn and D. Padua. Sagittal-Plane Trunk Position, Landing Forces, and Quadriceps Electromyographic Activity. Journal of Athletic Training, (2009) 44(2), pp.174-179.

[17] J. Chappell, D. Herman , B. Knight, D. Kirkendall, W. Garrett, and B. Yu. Effect of Fatigue on Knee Kinetics and Kinematics in Stop-Jump Tasks. The American Journal of Sports Medicine , (2005)33(7), pp.1022-1029.

[18] Peng L. Individual Choice and Reputation Distribution of Cooperative Behaviors among Heterogeneous Groups. Chaos, Solitons \& Fractals, 2015, 77: 39-46.

[19]Z. Lyons, G. Watt, Z. Shen, A. Janca. Acupuncture and Chinese herbs as treatments for depression, an Australian pilot study. Complement Therapies in Clinical Practice, 18(2012), pp216-220.

[20] M. Alexander, S. Paola, D. Helen. The management of cancer-related fatigue after chemotherapy with acupuncture and acupressure: A randomised controlled trial. Complementary Therapies in Medicine (2007)15,228-237

[21] M. Kim, J. Kim, H. Lee, A. Kim, H. Park and O. Kwon. Moxibustion for cancer-related fatigue: study protocol for a randomized controlled trial,BMC Complementary and Alternative Medicine (2017) 17:353

[22]I. Smilios. Effects of Varying Levels of Muscular Fatigue on Vertical Jump Performance. Journal of Strength and Conditioning Research, (1998) 12(3), pp.204-208.

[23]A. Nathaniel, R. Kevin, Ford, D .Gregory, Myer, E. Timothy. Impact differences in ground reaction force and center of mass between the first and second landing phases of a drop vertical jump and their implications for injury risk assessment. Journal of Biomechanics, 46 (2013)1237-1241 